\title{
The stability of the anisotropic parabolic equation with the variable exponent
}

Huashui Zhan*

\section{*Correspondence:}

huashuizhan@163.com

School of Applied Mathematics,

Xiamen University of Technology,

Xiamen, 361024, P.R. China

\section{Abstract}

Consider the equation

$$
u_{t}=\operatorname{div}\left(\overrightarrow{a(x)|\nabla u|^{p(x)-2} \nabla u}\right)=\sum_{i=1}^{N}\left(a_{i}(x)\left|u_{x_{i}}\right|^{p_{i}(x)-2} u_{x_{i}}\right)_{x^{\prime}}
$$

with $a_{i}(x), p_{i}(x) \in C^{1}(\bar{\Omega}), p_{i}(x)>1, a_{i}(x) \geq 0$. Basing on the weighted variable exponent Sobolev space, a new kind of weak solutions of the equation is introduced. Whether the usual Dirichlet homogeneous boundary value condition can be imposed depends on whether $a_{i}(x)$ is degenerate on the boundary or not. If some of $\left\{a_{i}(x)\right\}$ are degenerate on the boundary, a partial boundary value condition is imposed. If every $a_{i}(x)$ is degenerate on the boundary, by the new definition of a weak solution, the stability of weak solutions can be proved without any boundary value condition.

MSC: 35K55; 35K92; 35K85; 35R35

Keywords: anisotropic parabolic equation; boundary value condition; the stability

\section{Introduction}

In recent years, the parabolic equation with variable nonlinearities

$$
u_{t}=\operatorname{div}\left(|\nabla u|^{p(x)-2} \nabla u\right), \quad(x, t) \in Q_{T}=\Omega \times(0, T),
$$

coming from the so-called electrorheological fluids theory (see [1, 2]), has been researched widely [3-5]. Here, $\Omega \subset \mathbb{R}^{N}$ is a bounded domain with smooth boundary $\partial \Omega, p(x)>1$ is a measurable function. Recently, we have studied the equation

$$
u_{t}=\operatorname{div}\left(a(x)|\nabla u|^{p(x)-2} \nabla u\right), \quad(x, t) \in Q_{T},
$$

with

$$
\begin{aligned}
& u(x, 0)=u_{0}(x), \quad x \in \Omega, \\
& u(x, t)=0, \quad(x, t) \in \partial \Omega \times(0, T),
\end{aligned}
$$

in our previous works [6, 7]. Here $a(x) \in C(\bar{\Omega})$, and when $x \in \Omega, a(x)>0$. The main dedications of [6,7] are that, if $\left.a(x)\right|_{x \in \partial \Omega}=0$, then the stability of weak solutions can be proved

(c) The Author(s) 2017. This article is distributed under the terms of the Creative Commons Attribution 4.0 International License (http://creativecommons.org/licenses/by/4.0/), which permits unrestricted use, distribution, and reproduction in any medium, provided you give appropriate credit to the original author(s) and the source, provide a link to the Creative Commons license, and indicate if changes were made. 
without the boundary value condition, provided that the diffusion coefficient $a(x)$ satisfies some other restrictions.

In this paper, we consider an anisotropic parabolic equation of the type

$$
u_{t}=\operatorname{div}\left(\overrightarrow{a(x)|\nabla u|^{p(x)-2} \nabla u}\right)=\sum_{i=1}^{N}\left(a_{i}(x)\left|u_{x_{i}}\right|^{p_{i}(x)-2} u_{x_{i}}\right)_{x_{i}}, \quad(x, t) \in Q_{T},
$$

we denote that

$$
\begin{aligned}
& p_{0}=\min _{x \in \bar{\Omega}}\left\{p_{1}(x), p_{2}(x), \ldots, p_{N-1}(x), p_{N}(x)\right\}, \quad p_{0}>1, \\
& p^{0}=\max _{x \in \bar{\Omega}}\left\{p_{1}(x), p_{2}(x), \ldots, p_{N-1}(x), p_{N}(x)\right\} .
\end{aligned}
$$

We assume that $a_{i}(x) \in C(\bar{\Omega})$, and when $x \in \Omega, a_{i}(x)>0$. If for every $i=1,2, \ldots, N$,

$$
a_{i}(x)=0, \quad x \in \partial \Omega,
$$

then we can expect that similar conclusions as those in $[6,7]$ are true. While only some of $a_{i}(x)$ satisfy (1.6), the situations may be different. To see that, let us give a simple example to show the difference. Let $N=2, p_{1}(x)=p_{2}(x) \equiv p$, the domain $\Omega$ be a square,

$$
\Omega=\left\{\left(x_{1}, x_{2}\right): 0<x_{1}<1,0<x_{2}<1\right\} .
$$

Suppose that $a_{2}(x) \equiv 1$ and

$$
a_{1}(x)=0, \quad x \in \partial \Omega,
$$

consider the equation

$$
u_{t}=\frac{\partial}{\partial x_{1}}\left(a_{1}(x)\left|u_{x_{1}}\right|^{p-2} u_{x_{1}}\right)+\frac{\partial}{\partial x_{2}}\left(\left|u_{x_{1}}\right|^{p-2} u_{x_{2}}\right) .
$$

To obtain the stability of weak solutions of (1.8), initial value condition (1.3) is indispensable. However, instead of the usual Dirichlet boundary value condition (1.4), we may conjecture that only a partial boundary value condition

$$
u(x, t)=0, \quad(x, t) \in \Sigma_{2} \times(0, T),
$$

is required. Here

$$
\Sigma_{2}=\left\{\left(x_{1}, x_{2}\right): 0<x_{1}<1, x_{2}=0\right\} \cup\left\{\left(x_{1}, x_{2}\right): 0<x_{1}<1, x_{2}=1\right\} .
$$

By the way, we would like to suggest some of important works related to the equations

$$
\sum_{i=1}^{N}\left(a_{i}(x)\left|u_{x_{i}}\right|^{p_{i}(x)-2} u_{x_{i}}\right)=f(\lambda, u)
$$


with

$$
u=0, \quad x \in \partial \Omega,
$$

where $f(\lambda, u)$ appears in different forms in different papers. Di Nardo and Feo [8] gave the condition of the existence and uniqueness for problems (1.11)-(1.12); Mihailescu et al. [9] studied the usual eigenvalue problems. Later, Radulescu [10] studied the eigenvalue problems with sign-changing potential; Di Castro $[11,12]$ studied the regularity; El Hamidi and Vétois [13] studied the sharp Sobolev asymptotics; Vétois [14] studied the blow-up phenomena, Konaté and Ouaro [15] studied nonlinear anisotropic problems with bounded Radon diffuse measure and variable exponent and proved the existence and uniqueness of entropy solution. Also one can refer to some other related papers [16-26]. In a word, the elliptic anisotropic equations with the variable exponent have been given great attention recently, a lot of interesting results have been obtained. More or less beyond my imagination, there are few references related to the anisotropic parabolic equations with the variable exponent. We only have found one paper by Antontsev and Shmarev [27], in which the existence of weak solutions was studied when $a(x)=1$ in equation (1.5).

The most important characteristic of equation (1.5) lies in that the diffusions coefficients $a_{i}$ are different from one to another. After giving an existence result, the main aim of this paper is to give a complete classification of boundary value conditions and to study the corresponding stability of weak solutions.

\section{The definition of weak solutions}

Set

$$
C_{+}(\bar{\Omega})=\left\{h \in C(\bar{\Omega}): \min _{x \in \bar{\Omega}} h(x)>1\right\} .
$$

For any $h \in C_{+}(\bar{\Omega})$, we define

$$
h^{+}=\sup _{x \in \Omega} h(x), \quad h^{-}=\inf _{x \in \Omega} h(x)
$$

Let $a$ be a measurable positive and a.e. finite function in $\mathbb{R}^{N}$ satisfying

(C1) $a \in L_{\mathrm{loc}}^{1}(\Omega)$ and $a^{-\frac{1}{p(x)-1}} \in L_{\mathrm{loc}}^{1}(\Omega)$;

(C2) $a^{-s(x)} \in L^{1}(\Omega)$ with $s(x) \in\left(\frac{N}{p(x)}, \infty\right) \cap\left[\frac{1}{p(x)-1}, \infty\right)$.

For any $p \in C_{+}(\bar{\Omega})$, the definitions of the weighted variable exponent Lebesgue spaces $L^{p(x)}(a, \Omega)$ and the weighted variable exponent Sobolev spaces $W^{1, p(x)}(a, \Omega)$, and the following lemmas, can be found in [28].

\section{Lemma 2.1 Denote}

$$
\rho(u)=\int_{\Omega} a(x)|u|^{p(x)} d x \quad \text { for all } u \in L^{p(x)}(a, \Omega) .
$$

Then

(1) $\rho(u)>1(=1 ;<1)$ if and only if $\|u\|_{L^{p(x)}(a, \Omega)}>1(=1 ;<1)$, respectively;

(2) If $\|u\|_{L^{p(x)(a, \Omega)}}>1$, then $\|u\|_{L^{p(x)}(a, \Omega)}^{p^{-}} \leq \rho(u) \leq\|u\|_{L^{p(x)}(a, \Omega)}^{p^{+}}$; 
(3) If $\|u\|_{L^{p(x)}(a, \Omega)}<1$, then $\|u\|_{L^{p(x)}(a, \Omega)}^{p^{+}} \leq \rho(u) \leq\|u\|_{L^{p(x)}(a, \Omega)}^{p^{-}}$.

Lemma 2.2 If $1<p_{0} \leq p(x) \leq p_{1}<\infty$, then

$$
\begin{aligned}
& {\left[L^{p(x)}(a, \Omega)\right]^{*}=L^{p^{\prime}(x)}\left([a(x)]^{\frac{1}{1-p(x)}}, \Omega\right), \quad \frac{1}{p(x)}+\frac{1}{p^{\prime}(x)}=1,} \\
& \left|\int_{\Omega} u(x) v(x) d x\right| \leq k\|u\|_{L^{p^{\prime}(x)}\left([a(x)]^{\left.\frac{1}{1-p(x)}, \Omega\right)}\right.}\|v\|_{L^{p(x)}(a, \Omega)},
\end{aligned}
$$

where $\left[L^{p(x)}(a, \Omega)\right]^{*}$ is the conjugate space, $p^{\prime}(x)=\frac{p(x)-1}{p(x)}$.

Lemma 2.3 Let $p, s \in C_{+}(\bar{\Omega})$, and let $(\mathrm{C} 1)$ and $(\mathrm{C} 2)$ be satisfied. Then there exists the following compact embedding:

$$
W^{1, p(x)}(a, \Omega) \hookrightarrow \hookrightarrow L^{r(x)}(\Omega)
$$

provided that $r \in C_{+}(\bar{\Omega})$ and $1 \leq r(x)<p_{s}^{*}(x)$ for all $x \in \Omega$. Here,

$$
p_{s}(x)=\frac{p(x) s(x)}{1+s(x)}
$$

and

$$
p_{s}^{*}(x)= \begin{cases}\frac{p(x) s(x) N}{(s(x)+1) N-p(x) s(x)} & \text { if } p_{s}(x)<N \\ +\infty & \text { if } p_{s}(x) \geq N\end{cases}
$$

The basic definition is as follows.

Definition 2.4 A function $u(x, t)$ is said to be a weak solution of equation (1.5) with initial value condition (1.3) if

$$
u \in L^{\infty}\left(Q_{T}\right), \quad \frac{\partial u}{\partial t} \in L^{2}\left(Q_{T}\right), \quad u_{x_{i}} \in L^{\infty}\left(0, T ; L^{p_{i}(x)}\left(a_{i}, \Omega\right)\right),
$$

and for any function $\varphi \in L^{2}\left(0, T ; L^{p_{0}}(\Omega)\right),\left.\varphi\right|_{x \in \partial \Omega}=0, \varphi_{x_{i}} \in L^{2}\left(0, T ; L^{p_{i}(x)}\left(a_{i}, \Omega\right)\right)$ such that

$$
\iint_{Q_{T}}\left[\frac{\partial u}{\partial t} \varphi+\sum_{i=1}^{N} a_{i}(x)\left|u_{x_{i}}\right|^{p_{i}(x)-2} u_{x_{i}} \varphi_{x_{i}}\right] d x d t=0
$$

Initial value condition (1.3) is satisfied in the sense of

$$
\lim _{t \rightarrow 0} \int_{\Omega}\left|u(x, t)-u_{0}(x)\right| d x=0 .
$$

In this paper, we first study the existence of a weak solution.

Theorem 2.5 If $p_{i}(x)>1, a_{i}(x)$ satisfies conditions $(\mathrm{C} 1),(\mathrm{C} 2)$,

$$
u_{0} \in L^{\infty}(\Omega), \quad u_{0 x_{i}} \in L^{p_{i}(x)}\left(a_{i}, \Omega\right)
$$

then there is a solution of equation (1.5) with initial value (1.3). 
Theorem 2.6 If for every $1 \leq i \leq N, \int_{\Omega} a_{i}^{-\frac{1}{p_{i}(x)-1}}(x) d x<\infty$, then initial-boundary value problem (1.5)-(1.3)-(1.4) has a solution. Here, boundary value condition (1.4) is satisfied in the sense of trace.

Then we will study the stability of weak solutions according to the classification of boundary value conditions.

The first case is that we can impose the usual Dirichlet boundary value condition.

Theorem 2.7 If $u$ and $v$ are two solutions of equation (1.5) with the usual homogeneous value condition

$$
u(x, t)=v(x, t)=0, \quad(x, t) \in \partial \Omega \times(0, T),
$$

and with the initial values $u_{0}(x), v_{0}(x)$, respectively, then

$$
\int_{\Omega}|u(x, t)-v(x, t)| d x \leq \int_{\Omega}\left|u_{0}(x)-v_{0}(x)\right| d x .
$$

The second case is that we only can impose a partial boundary value condition. This case corresponds to that some of $\left\{a_{i}(x)\right\}$ are degenerate on the boundary, while others are not degenerate on the boundary. For simplicity, in this case, we assume that the domain $\Omega$ is just an $n$-dimensional cube

$$
\Omega=\left\{\left(x_{1}, x_{2}, \ldots, x_{N}\right): 0<x_{1}<1,0<x_{2}<1, \ldots, 0<x_{N}<1\right\} .
$$

Assume that $k+l=N, I=\left\{i_{1}, i_{2}, \ldots, i_{k}\right\} \subset\{1,2, \ldots, N\}, J=\left\{j_{1}, j_{2}, \ldots, j_{l}\right\} \subset\{1,2, \ldots, N\}, I \cap J=$ $\varnothing$, and

$$
\begin{array}{ll}
a_{i_{1}}(x)>0, a_{i_{2}}(x)>0, \ldots, a_{i_{k}}(x)>0, & x \in \bar{\Omega}, \\
a_{j_{1}}(x)>0, a_{j_{2}}(x)>0, \ldots, a_{j_{l}}(x)>0, & x \in \Omega .
\end{array}
$$

Instead of the usual Dirichlet boundary value condition (1.4), in this case, only a partial boundary value condition is imposed.

$$
u(x, t)=0, \quad(x, t) \in \Sigma \times(0, T)
$$

where

$$
\Sigma=\bigcup_{s=1}^{k}\left\{x \in \partial \Omega: x_{i_{s}}=0 \text { or } 1\right\} .
$$

Similar to the proof of Theorem 2.6, if

$$
\int_{\Omega} a_{s}^{-\frac{1}{p_{s}(x)-1}}(x) d x<\infty, \quad s=j_{1}, j_{2}, \ldots, j_{l},
$$

we can prove that there exists a solution of initial-boundary value problem (1.5)-(1.3)(2.10). 
Theorem 2.8 Besides (C1)-(C2), (2.8), (2.9) and (2.11), we assume that $\Omega$ satisfies (2.7),

$$
\begin{aligned}
& a_{j_{r}}(x)=a_{j_{r}}\left(x_{j_{r}}\right), \quad r=1,2, \ldots, l, \\
& a_{j_{r}}\left(x_{j_{r}}\right)=0, \quad x_{j_{r}}=0, \text { or } 1,
\end{aligned}
$$

and for large enough $n$,

$$
n^{1-\frac{1}{p_{j_{r}}^{+}}}\left(\left(\int_{0}^{\frac{1}{n}}+\int_{1-\frac{1}{n}}^{1}\right)\left|a_{j_{r}}(x)\right|^{p_{j_{r}}(x)} d x_{j_{r}}\right)^{\frac{1}{p_{r}^{+}}} \leq c .
$$

If $u$ and $v$ are two solutions of equation (1.5) with the same partial boundary value condition (2.10), and with the initial values $u_{0}(x), v_{0}(x)$, respectively, then

$$
\int_{\Omega}|u(x, t)-v(x, t)| d x \leq \int_{\Omega}\left|u_{0}(x)-v_{0}(x)\right| d x
$$

Certainly, we believe that, instead of conditions (2.12)-(2.13), only if

$$
a_{j_{r}}(x)=0, \quad x_{j_{r}}=0, \text { or } 1,
$$

the same conclusion of Theorem 2.8 is also true. But we cannot obtain this result for the time being.

The last case is that all $a_{i}(x)$ are degenerate on the boundary. Then we can prove the stability of weak solutions without any boundary value condition.

Theorem 2.9 If $a_{i}$ satisfies (C1)-(C2), and for large enough $n$,

$$
n^{1-\frac{1}{p_{i}^{+}}}\left(\int_{\Omega \backslash \Omega_{n}}\left|a_{i}(x)\right|^{p_{i}(x)} d x\right)^{\frac{1}{p_{i}^{+}}} \leq c
$$

let $u, v$ be two solutions of equation (1.5) with the initial values $u_{0}, v_{0}$, respectively. Then

$$
\int_{\Omega}|u(x, t)-v(x, t)| d x \leq \int_{\Omega}|u(x, 0)-v(x, 0)| d x
$$

where $\Omega_{n}=\left\{x \in \Omega: \prod_{i=1}^{N} a_{i}(x)>\frac{1}{n}\right\}$.

\section{The proof of Theorems 2.5-2.7}

Let $a(x)$ satisfy $(\mathrm{C} 1),(\mathrm{C} 2)$. Consider the regularized equation

$$
u_{t}=\operatorname{div}\left(\overrightarrow{a(x)|\nabla u|^{p(x)-2} \nabla u}\right)+\varepsilon \Delta u, \quad(x, t) \in Q_{T},
$$

with the initial boundary conditions

$$
\begin{aligned}
& u(x, 0)=u_{0}(x), \quad x \in \Omega, \\
& u(x, t)=0, \quad(x, t) \in \partial \Omega \times(0, T) .
\end{aligned}
$$


Proof of Theorem 2.5 Similar to [8], we can easily prove that there is a solution $u_{\varepsilon}$ of initialboundary value problem (3.1)-(3.3), and there is a constant $c$ only dependent on $\left\|u_{0}\right\|_{L^{\infty}(\Omega)}$ but independent of $\varepsilon$ such that

$$
\left\|u_{\varepsilon}\right\|_{L^{\infty}\left(Q_{T}\right)} \leq c, \quad\left\|u_{\varepsilon t}\right\|_{L^{2}\left(Q_{T}\right)} \leq c
$$

Multiplying (3.1) by $u_{\varepsilon}$ and integrating it over $Q_{T}$, we have

$$
\begin{aligned}
& \frac{1}{2} \int_{\Omega} u_{\varepsilon}^{2} d x+\sum_{i=1}^{n} \iint_{Q_{T}} a_{i}(x)\left|u_{\varepsilon x_{i}}\right|^{p_{i}(x)} d x d t+\varepsilon \iint_{Q_{T}}\left|\nabla u_{\varepsilon}\right|^{2} d x d t \\
& \quad=\frac{1}{2} \int_{\Omega} u_{0}^{2}(x) d x
\end{aligned}
$$

then

$$
\varepsilon \iint_{Q_{T}}\left|\nabla u_{\varepsilon}\right|^{2} d x \leq c
$$

and

$$
\sum_{i=1}^{N} \iint_{Q_{T}} a_{i}(x)\left|u_{\varepsilon x_{i}}\right|^{p_{i}(x)} d x d t \leq c .
$$

Hence, by (3.4), (3.6), (3.7), using Lemma 2.3, there exists a function $u$ and an $n$ dimensional vector $\vec{\zeta}=\left(\zeta_{1}, \ldots, \zeta_{n}\right)$ satisfying

$$
u \in L^{\infty}\left(Q_{T}\right), \quad \frac{\partial u}{\partial t} \in L^{2}\left(Q_{T}\right), \quad \zeta_{i} \in L^{1}\left(0, T ; L^{\frac{p_{i}(x)}{p_{i}(x)-1}}\left(a_{i}^{\frac{1}{1-p_{i}(x)}}, \Omega\right)\right),
$$

and $u_{\varepsilon} \rightarrow$ u a.e. $\in Q_{T}$,

$$
\begin{aligned}
& u_{\varepsilon} \rightarrow u, \quad \text { weakly star in } L^{\infty}\left(Q_{T}\right), \\
& u_{\varepsilon} \rightarrow u, \quad \text { in } L^{2}\left(0, T ; L_{\mathrm{loc}}^{r}(\Omega)\right), \\
& \frac{\partial u_{\varepsilon}}{\partial t} \rightarrow \frac{\partial u}{\partial t}, \quad \text { in } L^{2}\left(Q_{T}\right), \\
& \varepsilon \nabla u_{\varepsilon} \rightarrow 0, \quad \text { in } L^{2}\left(Q_{T}\right), \\
& a_{i}(x)\left|u_{\varepsilon x_{i}}\right|^{p_{i}(x)-2} u_{\varepsilon x_{i}} \rightarrow \zeta_{i}, \quad \text { in } L^{1}\left(0, T ; L^{\frac{p_{i}(x)}{p_{i}(x)-1}}\left(a_{i}^{\frac{1}{1-p_{i}(x)}}, \Omega\right)\right) .
\end{aligned}
$$

Here $r<\frac{N p_{0}}{N-p^{0}}$.

Now, similar to the general evolutionary $p$-Laplacian equation, we are able to prove that (the details are omitted here)

$$
\lim _{t \rightarrow 0} \int_{\Omega}\left|u(x, t)-u_{0}(x)\right| d x=0,
$$

and

$$
\sum_{i=1}^{N} \iint_{Q_{T}} a_{i}(x)\left|u_{x_{i}}\right|^{p_{i}(x)-2} u_{x_{i}} \varphi_{x_{i}} d x d t=\sum_{i=1}^{N} \iint_{Q_{T}} \zeta_{i}(x) \varphi_{x_{i}} d x d t,
$$


for any function $\varphi \in L^{2}\left(0, T ; L^{p_{0}}(\Omega)\right),\left.\varphi\right|_{x \in \partial \Omega}=0, \varphi_{x_{i}} \in L^{2}\left(0, T ; L^{p_{i}(x)}\left(a_{i}, \Omega\right)\right)$.

Then we have

$$
\iint_{Q_{T}}\left[\frac{\partial u}{\partial t} \varphi+\sum_{i=1}^{N} a_{i}(x)\left|u_{x_{i}}\right|^{p_{i}(x)-2} u_{x_{i}} \varphi_{x_{i}}\right] d x d t=0 .
$$

Thus $u$ satisfies equation (1.5) with initial value (1.3) in the sense of Definition 2.4.

Remark 3.1 In general, we only can obtain (3.8). It seems impossible to prove that, for every given $i$,

$$
\iint_{Q_{T}} a_{i}(x)\left|u_{x_{i}}\right|^{p_{i}(x)-2} u_{x_{i}} \varphi_{x_{i}} d x d t=\iint_{Q_{T}} \zeta_{i}(x) \varphi_{x_{i}} d x d t
$$

Lemma 3.2 Iffor any given $i \in\{1,2, \ldots, N\}, \int_{\Omega} a_{i}^{-\frac{1}{p_{i}(x)-1}}(x) d x<\infty$, then

$$
\int_{\Omega}\left|u_{x_{i}}\right| d x \leq c
$$

Proof

$$
\begin{aligned}
& \iint_{Q_{T}}\left|u_{x_{i}}\right| d x d t
\end{aligned}
$$

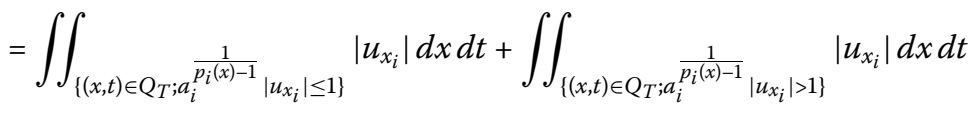

$$
\begin{aligned}
& \leq \iint_{Q_{T}} a^{-\frac{1}{p_{i}(x)-1}} d x d t+\iint_{Q_{\Omega}} a_{i}(x)\left|u_{x_{i}}\right|^{p_{i}(x)} d x d t \\
& \leq c \text {. }
\end{aligned}
$$

By this lemma, if for every $1 \leq i \leq N, \int_{\Omega} a_{i}^{-\frac{1}{p_{i}(x)-1}}(x) d x<\infty$, then

$$
\int_{\Omega}|\nabla u| d x \leq c
$$

Thus, we can define the trace of $u$ on the boundary $\partial \Omega$. Accordingly, Theorem 2.6 is obviously true.

Now, we will prove Theorem 2.7. For any given positive integer $n$, let $g_{n}(s)$ be an odd function, and

$$
g_{n}(s)= \begin{cases}1, & s>\frac{1}{n}, \\ n^{2} s^{2} \mathrm{e}^{1-n^{2} s^{2}}, & 0 \leq s \leq \frac{1}{n} .\end{cases}
$$

Clearly,

$$
\lim _{n \rightarrow 0} g_{n}(s)=\operatorname{sgn}(s), \quad s \in(-\infty,+\infty)
$$


Proof of Theorem 2.7 Let $u$ and $v$ be two weak solutions of equation (1.5) with the same boundary value condition (2.5) and with the initial values $u(x, 0), v(x, 0)$, respectively. By a process of limit, since (2.5) $u=v$ on the boundary, we can choose $\varphi=g_{n}(u-v)$ as the test function, then

$$
\begin{aligned}
& \int_{\Omega} g_{n}(u-v) \frac{\partial(u-v)}{\partial t} d x \\
& \quad+\sum_{i=1}^{N} \int_{\Omega} a_{i}(x)\left(\left|u_{x_{i}}\right|^{p_{i}(x)-2} u_{x_{i}}-\left|v_{x_{i}}\right|^{p_{i}(x)-2} v_{x_{i}}\right)\left(u_{x_{i}}-v_{x_{i}}\right) g_{n}^{\prime}(u-v) d x \\
& \quad=0
\end{aligned}
$$

Thus

$$
\begin{aligned}
& \lim _{n \rightarrow \infty} \int_{\Omega} g_{n}(u-v) \frac{\partial(u-v)}{\partial t} d x=\frac{d}{d t}\|u-v\|_{L^{1}(\Omega)}, \\
& \int_{\Omega} a_{i}(x)\left(\left|u_{x_{i}}\right|^{p_{i}(x)-2} u_{x_{i}}-\left|v_{x_{i}}\right|^{p_{i}(x)-2} v_{x_{i}}\right)\left(u_{x_{i}}-v_{x_{i}}\right) g_{n}^{\prime}(u-v) d x \geq 0 .
\end{aligned}
$$

Now, let $n \rightarrow \infty$ in (3.13). Then

$$
\frac{d}{d t}\|u-v\|_{L^{1}(\Omega)} \leq 0
$$

It implies that

$$
\int_{\Omega}|u(x, t)-v(x, t)| d x \leq \int_{\Omega}\left|u_{0}(x)-v_{0}(x)\right| d x, \quad \forall t \in[0, T) .
$$

\section{The stability of the partially boundary value conditions}

Proof of Theorem 2.8 Let $u$ and $v$ be two weak solutions of equation (1.5) with the initial values $u(x, 0), v(x, 0)$, respectively.

For $1 \leq r \leq l$, let

$$
\phi_{n, j_{r}}\left(x_{j_{r}}\right)= \begin{cases}1 & \text { if } x_{j_{r}}>\frac{1}{n}, \text { or } 1-x_{j_{r}}>\frac{1}{n} \\ n a_{j_{r}}\left(x_{j_{r}}\right) & \text { if } x_{j_{r}} \leq \frac{1}{n}, \text { or } 1-x_{j_{r}} \leq \frac{1}{n}\end{cases}
$$

By (2.10), (2.12), (2.13), $\prod_{r=1}^{l} \phi_{n, j_{r}}\left(x_{j_{r}}\right) g_{n}(u-v)(x)=0$ when $x \in \partial \Omega$, we can choose $\prod_{r=1}^{l} \phi_{n, j_{r}}\left(x_{j_{r}}\right) g_{n}(u-v)$ as the test function, then

$$
\begin{aligned}
& \int_{\Omega} \prod_{r=1}^{l} \phi_{n, j_{r}} g_{n}(u-v) \frac{\partial(u-v)}{\partial t} d x \\
& \quad+\sum_{i=1}^{N} \int_{\Omega} a_{i}(x)\left(\left|u_{x_{i}}\right|^{p_{i}(x)-2} u_{x_{i}}-\left|v_{x_{i}}\right|^{p_{i}(x)-2} v_{x_{i}}\right) \cdot\left(u_{x_{i}}-v_{x_{i}}\right) g_{n}^{\prime}(u-v) \prod_{r=1}^{l} \phi_{n, j_{r}} d x \\
& \quad+\sum_{i=1}^{N} \int_{\Omega} a_{i}(x)\left(\left|u_{x_{i}}\right|^{p_{i}(x)-2} u_{x_{i}}-\left|v_{x_{i}}\right|^{p_{i}(x)-2} v_{x_{i}}\right) g_{n}(u-v)\left(\prod_{r=1}^{l} \phi_{n, j_{r}}\right)_{x_{i}} d x=0 .
\end{aligned}
$$


Chan Boundary Value Problems (2017) 2017:134

Page 10 of 14

Thus

$$
\lim _{n \rightarrow \infty} \int_{\Omega} \prod_{r=1}^{l} \phi_{n, j_{r}} g_{n}(u-v) \frac{\partial(u-v)}{\partial t} d x=\frac{d}{d t}\|u-v\|_{L^{1}(\Omega)}
$$

and

$$
\int_{\Omega} a_{i}(x)\left(\left|u_{x_{i}}\right|^{p_{i}(x)-2} u_{x_{i}}-\left|v_{x_{i}}\right|^{p_{i}(x)-2} v_{x_{i}}\right) \cdot\left(u_{x_{i}}-v_{x_{i}}\right) g_{n}^{\prime}(u-v) \prod_{r=1}^{l} \phi_{n, j_{r}} d x \geq 0 .
$$

Moreover, since $\phi_{n, j_{r}}\left(x_{j_{r}}\right)$ is only dependent on the variable $x_{j_{r}}$, we have

$$
\begin{aligned}
& \sum_{i=1}^{N} \int_{\Omega} a_{i}(x)\left(\left|u_{x_{i}}\right|^{p_{i}(x)-2} u_{x_{i}}-\left|v_{x_{i}}\right|^{p_{i}(x)-2} v_{x_{i}}\right) g_{n}(u-v)\left(\prod_{r=1}^{l} \phi_{n, j_{r}}\right)_{x_{i}} d x \\
& =\sum_{s=1}^{k} \int_{\Omega} a_{i_{s}}(x)\left(\left|u_{x_{i_{s}}}\right|^{p_{i_{s}}(x)-2} u_{x_{i_{s}}}-\left|v_{x_{i_{s}}}\right|^{p_{i_{s}}(x)-2} v_{x_{i_{s}}}\right) g_{n}(u-v)\left(\prod_{r=1}^{l} \phi_{n, j_{r}}\right)_{x_{i_{s}}} d x \\
& \quad+\sum_{r=1}^{l} \int_{\Omega} a_{j_{r}}(x)\left(\left|u_{x_{j r}}\right|^{p_{j_{r}}(x)-2} u_{x_{j_{r}}}-\left|v_{x_{j_{r}}}\right|^{p_{j_{r}}(x)-2} v_{x_{j_{r}}}\right) g_{n}(u-v)\left(\prod_{r=1}^{l} \phi_{n, j_{r}}\right)_{x_{j_{r}}} d x \\
& =\sum_{r=1}^{l} \int_{\Omega} a_{j_{r}}(x)\left(\left|u_{x_{j_{r}}}\right|_{p_{j_{r}}(x)-2} u_{x_{j_{r}}}-\left|v_{x_{j_{r}}}\right|^{p_{j_{r}}(x)-2} v_{x_{j_{r}}}\right) g_{n}(u-v)\left(\prod_{r=1}^{l} \phi_{n, j_{r}}\right)_{x_{j_{r}}} d x .
\end{aligned}
$$

Clearly,

$$
\left(\prod_{r=1}^{l} \phi_{n, j_{r}}\right)_{x_{j_{1}}}=\prod_{r=1, r \neq r_{1}}^{l} \phi_{n, j_{r}} \phi_{n, j_{r_{1}}}^{\prime}\left(x_{j_{r_{1}}}\right)
$$

and $\left|\phi_{n, j_{r}}^{\prime}\left(x_{j_{r}}\right)\right|=n a_{j_{r}}^{\prime}\left(x_{j_{r}}\right)$ when $x_{j_{r}}<\frac{1}{n}$ or $1-x_{j_{r}}<\frac{1}{n}$, in other places, it is identical to zero.

Accordingly, for any $j_{r_{1}} \in\left\{j_{1}, j_{2}, \ldots, j_{l}\right\}$, if we notice that

$$
\Omega=(0,1) \times(0,1) \times \cdots \times(0,1),
$$

by conditions (2.12)-(2.13), we have

$$
\begin{aligned}
& \left|\int_{0}^{1} a_{j_{r_{1}}}(x)\left(\left|u_{x_{j_{1}}}\right|^{p_{j_{r_{1}}}(x)-2} u_{x_{j_{1}}}-\left|v_{x_{j_{1}}}\right|^{p_{r_{1}}(x)-2} v_{x_{j_{1}}}\right) g_{n}(u-v)\left(\prod_{r=1}^{l} \phi_{n, j_{r}}\right)_{x_{j_{1}}} d x_{j_{r_{1}}}\right| \\
& \quad=\mid\left(\int_{0}^{\frac{1}{n}}+\int_{\frac{1}{n}}^{1}\right) a_{j_{r_{1}}}\left(x_{j_{r_{1}}}\right)\left(\left|u_{x_{j_{1}}}\right|^{p_{j_{r_{1}}}(x)-2} u_{x_{j_{1}}}-\left|v_{x_{j}}\right|^{p_{j_{1}}}(x)-2 v_{x_{r_{1}}}\right) g_{n}(u-v) \\
& \quad \times \prod_{r=1, r \neq r_{1}}^{l} \phi_{n, j_{r_{1}}} \phi_{n, j_{r_{1}}}^{\prime}\left(x_{j_{r_{1}}}\right) d x_{j_{r_{1}}} \mid \\
& \quad \leq\left. c n\left(\int_{0}^{\frac{1}{n}}+\int_{\frac{1}{n}}^{1}\right) a_{j_{r_{1}}}\left(x_{j_{r_{1}}}\right)|| u_{x_{j_{1}}}\right|^{p_{j_{r_{1}}}(x)-2} u_{x_{j_{1}}}-\left|v_{x_{j_{r}}}\right|^{p_{r_{r_{1}}}(x)-2} v_{x_{j_{r_{1}}}}|| a_{j_{r_{1}}}^{\prime}\left(x_{j_{r_{1}}}\right) \mid d x_{j_{r_{1}}}
\end{aligned}
$$




$$
\begin{aligned}
& \leq c n\left\|\left(\left|u_{x_{j_{1}}}\right|^{p_{r_{r_{1}}}(x)-1}+\left|\nabla v_{x_{j_{1}}}\right|^{p_{r_{r_{1}}}(x)-1}\right)\right\|_{L^{q_{j_{r_{1}}}}(x)\left(\left[a_{j_{1}}\left(x_{r_{1}}\right)\right]^{\frac{1}{1-p_{j_{1}}(x)}},\left(0, \frac{1}{n}\right) \cup\left(1-\frac{1}{n}, 1\right)\right)}
\end{aligned}
$$

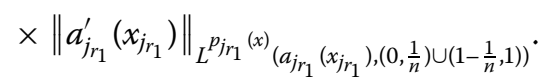

By Lemma 2.2, we have

$$
\begin{aligned}
& n\left\|a_{j_{r_{1}}}^{\prime}\left(x_{j_{r_{1}}}\right)\right\|_{L^{p_{r_{1}}}}(x)\left(a_{j_{r_{1}}}\left(x_{j_{r_{1}}}\right),\left(0, \frac{1}{n}\right) \cup\left(1-\frac{1}{n}, 1\right)\right) \\
& \quad \leq n\left(\left(\int_{0}^{\frac{1}{n}}+\int_{\frac{1}{n}}^{1}\right) a_{j_{r_{1}}}\left(x_{j_{r_{1}}}\right)\left|a_{j_{r_{1}}}^{\prime}\left(x_{j_{r_{1}}}\right)\right|^{p_{j_{r_{1}}}(x)} d x\right)^{\frac{1}{p_{r_{1}}^{+}}} \\
& \quad \leq c n^{1-\frac{1}{p_{r_{1}}^{+}}}\left(\left(\int_{0}^{\frac{1}{n}}+\int_{\frac{1}{n}}^{1}\right)\left|a_{j_{r_{1}}}^{\prime}\left(x_{j_{r_{1}}}\right)\right|^{p_{j_{r_{1}}}(x)} d x\right)^{\frac{1}{p_{r_{1}}^{+}}} \leq c .
\end{aligned}
$$

Then, by (4.5)-(4.6), we have

$$
\begin{aligned}
& \mid \int_{0}^{1} a_{j_{r_{1}}}(x)\left(\left|u_{x_{j_{1}}}\right|^{p_{r_{1}}(x)-2} u_{x_{j_{1}}}-\left|v_{x_{j_{1}}}\right|_{j_{r_{1}}(x)-2} v_{x_{j_{r_{1}}}}\right) \\
& \quad \times(u-v) g_{n}(u-v)\left(\prod_{r=1}^{l} \phi_{n, j_{r}}\right)_{x_{j_{r_{1}}}} d x_{j_{r_{1}}} \mid \\
& \quad \leq c\left(\left(\int_{0}^{\frac{1}{n}}+\int_{\frac{1}{n}}^{1}\right) a_{j_{r_{1}}}\left(x_{j_{r_{1}}}\right)\left|u_{x_{r_{1}}}\right|^{p_{r_{1}}(x)} d x_{j_{r_{1}}}\right)^{\frac{1}{q_{r_{1}}^{+}}} \\
& \quad+c\left(\left(\int_{0}^{\frac{1}{n}}+\int_{\frac{1}{n}}^{1}\right) a_{j_{r_{1}}}\left(x_{j_{r_{1}}}\right)\left|v_{x_{j_{1}}}\right|^{p_{r_{1}}(x)} d x_{j_{r_{1}}}\right)^{\frac{1}{q_{j_{1}}^{+}}}
\end{aligned}
$$

which goes to 0 as $n \rightarrow \infty$.

Now, let $n \rightarrow \infty$ in (4.1). Then

$$
\frac{d}{d t}\|u-v\|_{L^{1}(\Omega)} \leq 0
$$

It implies that

$$
\int_{\Omega}|u(x, t)-v(x, t)| d x \leq \int_{\Omega}\left|u_{0}-v_{0}\right| d x, \quad \forall t \in[0, T)
$$

\section{The stability without the boundary value condition}

Proof of Theorem 2.9 Let $u$ and $v$ be two weak solutions of equation (1.5) with the initial values $u(x, 0)$ and $v(x, 0)$, respectively, but without any boundary value condition.

Let $\Omega_{n}=\left\{x \in \Omega: \prod_{i=1}^{N} a_{i}(x)>\frac{1}{n}\right\}$, and

$$
\phi_{n}(x)= \begin{cases}1 & \text { if } x \in \Omega_{n}, \\ n \prod_{i=1}^{N} a_{i}(x) & \text { if } x \in \Omega \backslash \Omega_{n} .\end{cases}
$$


We can choose $\chi_{[\tau, s]} \phi_{n} g_{n}(u-v)$ as the test function, where $\chi_{[\tau, s]}$ is the characteristic function of $[\tau, s) \subseteq[0, T)$, then

$$
\begin{aligned}
& \int_{\tau}^{s} \int_{\Omega} \phi_{n} g_{n}(u-v) \frac{\partial(u-v)}{\partial t} d x d t \\
& \quad+\sum_{i=1}^{N} \int_{\tau}^{s} \int_{\Omega} a_{i}(x)\left(\left|u_{x_{i}}\right|^{p_{i}(x)-2} u_{x_{i}}-\left|v_{x_{i}}\right|^{p_{i}(x)-2} v_{x_{i}}\right)\left(u_{x_{i}}-v_{x_{i}}\right) g_{n}^{\prime}(u-v) \phi_{n}(x) d x d t \\
& \quad+\sum_{i=1}^{N} \int_{\tau}^{s} \int_{\Omega} a_{i}(x)\left(\left|u_{x_{i}}\right|^{p_{i}(x)-2} u_{x_{i}}-\left|v_{x_{i}}\right|^{p_{i}(x)-2} v_{x_{i}}\right)\left(u_{x_{i}}-v_{x_{i}}\right) g_{n}(u-v) \phi_{n x_{i}} d x d t \\
& =0 .
\end{aligned}
$$

As usual, we have

$$
\int_{\Omega} a_{i}(x)\left(\left|u_{x_{i}}\right|^{p_{i}(x)-2} u_{x_{i}}-\left|v_{x_{i}}\right|^{p_{i}(x)-2} v_{x_{i}}\right)\left(u_{x_{i}}-v_{x_{i}}\right) g_{n}^{\prime}(u-v) \phi_{n}(x) d x \geq 0 .
$$

At the same time, since $u_{t} \in L^{2}\left(Q_{T}\right)$, using the Lebesgue dominated theorem, we have

$$
\begin{gathered}
\lim _{n \rightarrow \infty} \int_{\tau}^{s} \int_{\Omega} \phi_{n}(x) g_{n}(u-v) \frac{\partial(u-v)}{\partial t} d x d t \\
\quad=\int_{\Omega}|u-v|(x, s) d x-\int_{\Omega}|u-v|(x, \tau) d x .
\end{gathered}
$$

Thus, we only need to deal with the last term on the left-hand side of (5.2). Obviously, $\phi_{n x_{i}}=n\left(\prod_{j=1}^{N} a_{j}(x)\right)_{x_{i}}$ when $x \in \Omega \backslash \Omega_{n}$, in other places, it is identical to zero. By condition (2.15), we have

$$
\begin{aligned}
& \left|\int_{\Omega} a_{i}(x)\left(\left|u_{x_{i}}\right|^{p_{i}-2} u_{x_{i}}-\left|v_{x_{i}}\right|^{p_{i}-2} v_{x_{i}}\right) \phi_{\eta x_{i}} g_{n}(u-v) d x\right| \\
& =\left|\int_{\Omega \backslash \Omega_{n}} a_{i}(x)\left(\left|u_{x_{i}}\right|^{p_{i}(x)-2} u_{x_{i}}-\left|v_{x_{i}}\right|^{p_{i}(x)-2} v_{x_{i}}\right) \phi_{n x_{i}} g_{n}(u-v) d x\right| \\
& \leq n \int_{\Omega \backslash \Omega_{n}} a_{i}(x)\left(\left|u_{x_{i}}\right|^{p_{i}(x)-1}+\left|v_{x_{i}}\right|^{p_{i}(x)-1}\right)\left|\left(\prod_{j=1}^{N} a_{j}(x)\right)_{x_{i}} g_{n}(u-v)\right| d x \\
& \leq c n\left(\int_{\Omega \backslash \Omega_{n}} a_{i}(x)\left(\left|u_{x_{i}}\right|^{p_{i}(x)}+\left|v_{x_{i}}\right|^{p_{i}(x)}\right) d x\right)^{\frac{1}{q_{i}^{+}}}\left(\left.\int_{\Omega \backslash \Omega_{n}} a_{i}(x)\left|\left(\prod_{j=1}^{N} a_{j}(x)\right)_{x_{i}}\right|\right|^{p_{i}(x)} d x\right)^{\frac{1}{p_{i}^{+}}} \\
& \leq c\left[\left(\int_{\Omega \backslash \Omega_{n}} a_{i}(x)\left|u_{x_{i}}\right|^{p_{i}(x)} d x\right)^{\frac{1}{q_{i}^{+}}}+\left(\int_{\Omega \backslash \Omega_{n}} a_{i}(x)\left|v_{x_{i}}\right|^{p_{i}(x)} d x\right)^{\frac{1}{q_{i}^{+}}}\right] \\
& \quad \times\left[\frac{1}{\eta}\left(\int_{\Omega \backslash \Omega_{n}} a_{i}(x) \mid\left(\prod_{j=1}^{N} a_{j}(x)\right)_{x_{i}}^{p_{i}(x)} d x\right)^{\frac{1}{p_{i}^{+}}}\right] \\
& \quad \leq c\left(\int_{\Omega \backslash \Omega_{n}} a_{i}(x)\left|u_{x_{i}}\right|^{p_{i}(x)} d x\right)^{\frac{1}{q_{i}^{+}}}+c\left(\int_{\Omega \backslash \Omega_{n}} a_{i}(x)\left|v_{x_{i}}\right|^{p_{i}(x)} d x\right)^{\frac{1}{q_{i}^{+}}} .
\end{aligned}
$$

Here $q_{i}(x)=\frac{p_{i}(x)}{p_{i}(x)-1}, q_{i}^{+}=\max _{x \in \bar{\Omega}} q_{i}(x)$. 
Then

$$
\begin{aligned}
& \lim _{\eta \rightarrow 0}\left|\int_{\tau}^{s} \int_{\Omega} a_{i}(x)\left(\left|u_{x_{i}}\right|^{p_{i}(x)-2} u_{x_{i}}-\left|v_{x_{i}}\right|^{p_{i}(x)-2} v_{x_{i}}\right) \phi_{n x_{i}} g_{n}(u-v) d x d t\right| \\
& \quad \leq c \lim _{\eta \rightarrow 0}\left[\left(\int_{\Omega \backslash \Omega_{n}} a_{i}(x)\left|u_{x_{i}}\right|^{p_{i}(x)} d x\right)^{\frac{1}{q_{i}^{+}}}+\left(\int_{\Omega \backslash \Omega_{n}} a_{i}(x)\left|v_{x_{i}}\right|^{p_{i}(x)} d x\right)^{\frac{1}{q_{i}^{+}}}\right]=0 .
\end{aligned}
$$

Now, let $\eta \rightarrow 0$ in (5.2). Then

$$
\int_{\Omega}|u(x, s)-v(x, s)| d x \leq \int_{\Omega}|u(x, \tau)-v(x, \tau)| d x
$$

by the arbitrariness of $\tau$, we have

$$
\int_{\Omega}|u(x, s)-v(x, s)| d x \leq \int_{\Omega}\left|u_{0}(x)-v_{0}(x)\right| d x .
$$

\section{Conclusion}

Different from the usual evolutionary $p(x)$-Laplacian equation, the anisotropic parabolic equation is considered in this paper. Due to the anisotropic character, it admits that there are different diffusion coefficients corresponding to different directions. If in some directions the diffusion coefficients are degenerate on the boundary, while in other directions they are not degenerate, how to give a suitable partial boundary value condition to match the equation is a very interesting problem. The paper first researches this problem. By introducing a new kind of weak solutions, which are based on the weighted variable exponent Sobolev spaces, the existence and stability of the weak solutions are proved in this paper.

\section{Acknowledgements}

The paper is supported by NSF of Fujian Province (Grant No. 2015J1092), supported by SF of Xiamen University of Technology (Grant No. XYK201448), China.

\section{Competing interests}

The author declares that they have no competing interests.

\section{Authors' contributions}

All authors read and approved the final manuscript.

\section{Publisher's Note}

Springer Nature remains neutral with regard to jurisdictional claims in published maps and institutional affiliations.

Received: 28 July 2017 Accepted: 6 September 2017 Published online: 15 September 2017

\section{References}

1. Ruzicka, M: Electrorheological Fluids: Modeling and Mathematical Theory. Lecture Notes in Math., vol. 1748. Springer, Berlin (2000)

2. Acerbi, E, Mingione, G: Regularity results for stationary electrorheological fluids. Arch. Ration. Mech. Anal. 164, 213-259 (2002)

3. Antontsev, S, Shmarev, S: Anisotropic parabolic equations with variable nonlinearity. Publ. Mat. 53, 355-399 (2009)

4. Antontsev, S, Shmarev, S: Parabolic equations with double variable nonlinearities. Math. Comput. Simul. 81 2018-2032 (2011)

5. Lian, S, Gao, W, Yuan, H, Cao, C: Existence of solutions to an initial Dirichlet problem of evolutional $p(x)$-Laplace equations. Ann. Inst. Henri Poincaré, Anal. Non Linéaire 29, 377-399 (2012)

6. Zhan, $\mathrm{H}$, Wen, J: Evolutionary $p(x)$-Laplacian equation free from the limitation of the boundary value. Electron. J. Differ. Equ. 2016, 143 (2016)

7. Zhan, H: The stability of evolutionary p(x)-Laplacian equation. Bound. Value Probl. 2017, 13 (2017). doi:10.1186/s13661-016-0742-0 
8. Nardo, RD, Feo, F: Existence and uniqueness for nonlinear anisotropic elliptic equations. Arch. Math. 102, 141-153 (2014)

9. Mihailescu, M, Pucci, $P$, Radulescu, V: Eigenvalue problems for anisotropic quasilinear elliptic equations with variable exponent. J. Math. Anal. Appl. 340, 687-698 (2008)

10. Radulescu, V: Nonlinear elliptic equations with variable exponent: old and new. Nonlinear Anal. TMA 121, 336-369 (2015)

11. Di Castro, A: Anisotropic elliptic problems with natural growth terms. Manuscr. Math. 135, 521-543 (2011)

12. Di Castro, A: Local Holder continuity of weak solutions for an anisotropic elliptic equation. Nonlinear Differ. Equ. Appl. 20, 463-486 (2013)

13. El Hamidi, A, Vétois, J: Sharp Sobolev asymptotics for critical anisotropic equations. Arch. Ration. Mech. Anal. 192, $1-26(2009)$

14. Vétois, J: The blow-up of critical anisotropic equations with critical directions. Nonlinear Differ. Equ. Appl. 18, 173-197 (2011)

15. Konaté, I, Ouaro, S: Good Radon measure for anisotropic problems with variable exponent. Electron. J. Differ. Equ. 2016, 221 (2016)

16. Rădulescu, VD, Stancu, I-L: Combined concave-convex effects in anisotropic elliptic equations with variable exponents. Nonlinear Differ. Equ. Appl. 22(3), 391-410 (2015)

17. Fragalà, I, Gazzola, F, Kawohl, B: Existence and nonexistence results for anisotropic quasilinear equations. Ann. Inst. Henri Poincaré, Anal. Non Linéaire 21, 715-734 (2004)

18. Fu, F, Shan, Y: On the removability of isolated singular points for elliptic equations involving variable exponent. Adv. Nonlinear Anal. 5, 121-132 (2016)

19. Radulescu, $V$, Repovš, D: Combined effects in nonlinear problems arising in the study of anisotropic continuous media. Nonlinear Anal. 75, 1524-1530 (2012)

20. Radulescu, V, Repovš, D: Partial Differential Equations with Variable Exponents: Variational Methods and Qualitative Analysis. CRC Press, Boca Raton (2015)

21. Mihailescu, M, Radulescu, $V$, Repovš, D: On a non-homogeneous eigenvalue problem involving a potential: an Orlicz-Sobolev space setting. J. Math. Pures Appl. 93(2), 132-148 (2010)

22. Repovš, D: Stationary waves of Schrödinger-type equations with variable exponent. Anal. Appl. 13, 645-661 (2015)

23. Boureanu, M, Radulescu, V, Repovš, D: On a $p(\cdot)$-biharmonic problem with no-flux boundary condition. Comput. Math. Appl. 72, 2505-2515 (2016)

24. Alsaedi, R: Perturbed subcritical Dirichlet problems with variable exponents. Electron. J. Differ. Equ. 2016, 295 (2016)

25. Li, Z, Yan, B, Gao, W: Existence of solutions to a parabolic $p(x)$-Laplace equation with convection term via L-infinity estimates. Electron. J. Differ. Equ. 2015, 46 (2015)

26. Giacomoni, J, Radulescu, V, Warnault, G: Quasilinear parabolic problem with variable exponent: qualitative analysis and stabilization. Commun. Contemp. Math. (in press). doi:10.1142/S0219199717500651

27. Antontsev, S, Shmarev, S: Existence and uniqueness for doubly nonlinear parabolic equations with nonstandard growth conditions. Differ. Equ. Appl. 4(1), 67-94 (2012)

28. Ho, K, Sim, I: On degenerate $p(x)$-Laplacian equations involving critical growth with two parameters. Nonlinear Anal. $132,95-114(2016)$

\section{Submit your manuscript to a SpringerOpen ${ }^{\circ}$ journal and benefit from:}

- Convenient online submission

- Rigorous peer review

- Open access: articles freely available online

- High visibility within the field

- Retaining the copyright to your article

Submit your next manuscript at $\boldsymbol{\nabla}$ springeropen.com 